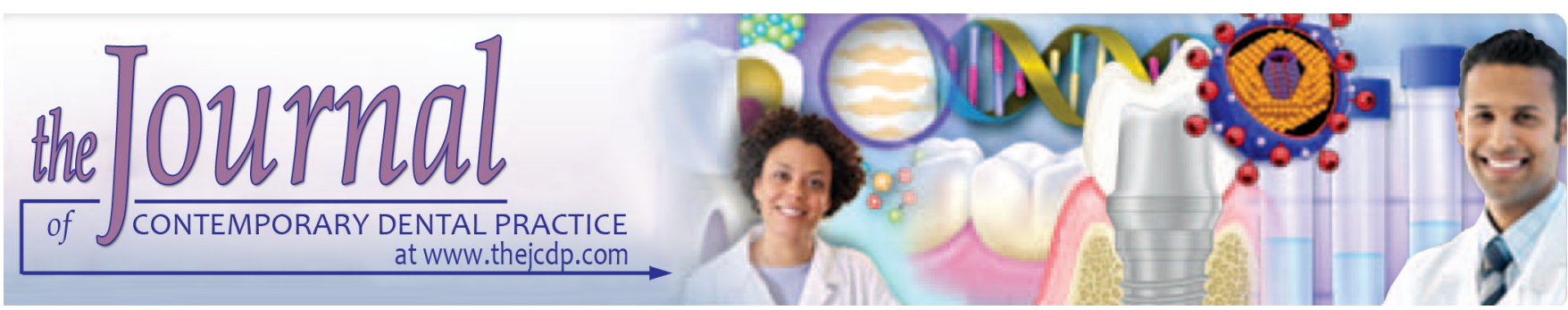

\title{
An In Vitro Study of Bactericidal Effect of Gallium Aluminium Arsenide Laser on Anaerobic Photosensitized Periodontopathics
}

\author{
${ }^{1}$ Kiran Dodani, ${ }^{2}$ Neha Khare, ${ }^{3}$ Chandrahas Bathini, ${ }^{4}$ Supriya Mishra, ${ }^{5}$ Mohammed NK Inamdar, ${ }^{6}$ Amit Nasha
}

\begin{abstract}
Aim: To investigate the bactericidal effect of gallium aluminium arsenide (GaAIAs) laser on photosensitized predominant periodontopathogenic anaerobic organisms.

Materials and methods: The present prospective study was carried out among 50 patients of chronic periodontitis with pockets $\geq 5 \mathrm{~mm}$ depth. For sample analysis of $P$. intermedia and $P$. gingivalis, a subgingival plaque was collected from periodontal pockets using sterile curettes under aseptic conditions. The sample was then immediately transported to microbiology laboratory using Transport media, Thioglycollate broth with Vitamin $\mathrm{K}$ and Hemin. After incubation period again the number of the viable bacterial count was carried out using a magnifying glass and expressed as CFU/mL, to determine the bactericidal effect of GaAlAs laser. A predetermined number of colonies (for $P$. intermedia 500 colonies were taken initially; for $P$. gingivalis 400 colonies were taken initially) were taken equally as control group and case group. The data was statistically analyzed using the SPSS statistical software version 16. Mann-Whitney Test was used for statistical analysis with $p$ value less than 0.05 considered statically significant.
\end{abstract}

Results: Study group shows a reduction in colony count of $P$. intermedia after being exposed to GaAlAs diode laser for 1 minute from 369-229, 134 and 41 which was statistically significant with $p$ value $<0.0001$. Study group shows a decrease in colony count of $P$. gingivalis after being exposed to GaAlAs

\footnotetext{
1,2,6 Department of Periodontics, RKDF Dental College and Research Centre, Bhopal, Madhya Pradesh, India

${ }^{3}$ Department of Periodontics, Hedgewar Smruti Rugna Seva Mandal Dental College, Hingoli, Maharashtra, India

${ }^{4}$ Department of Periodontics, Government Dental College, Raipur, Chhattisgarh, India

${ }^{5}$ Department of Periodontics, Preventive Dental Sciences, College of Pharmacy and Dentistry, Buraydah, Kingdom of Saudi Arabia

Corresponding Author: Kiran Dodani, Department of Periodontics, RKDF Dental College and Research Centre, Bhopal, Madhya Pradesh, India, Phone: +917737732117, e-mail: drkasturba79@yahoo.co.in
}

diode laser for 1 minute from 286.52-197, 94 and 39 which was statistically significant with $p$ value $<0.0001$.

Conclusion: The result of our study implies that for all tested bacterial strains, the effect of GaAIAs laser for 1 minute resulted in a significant reduction in the viable counts of photosensitized predominant periodontopathogenic anaerobic organisms. Photodynamic therapy (PDT) kills the bacteria and also leads to the detoxification of endotoxins. Further, it can thus be safely concluded that this technique is an alternative method adjunct to mechanical therapy.

Clinical significance: The application of an alternative method to eradicate bacteria from periodontal pockets is desirable. One such approach is photodynamic therapy (PDT), i.e., laser therapy. Owing to the emergence of antibiotic resistance, PDT has to turn out to be a feasible alternative antibacterial therapy for biofilm-related diseases.

Keywords: Diode laser, Periodontopathogens, Photodynamic therapy, $P$. gingivalis, $P$. intermedia.

How to cite this article: Dodani K, Khare N, Bathini C, Mishra S, Inamdar MNK, Nasha A. An In Vitro Study of Bactericidal Effect of Gallium Aluminium Arsenide Laser on Anaerobic Photosensitized Periodontopathics. J Contemp Dent Pract 2019;20(3):385-389.

Source of support: Nil

Conflict of interest: None

\section{INTRODUCTION}

The currently considered key Gram-negative periodontopathogens include Porphyromonas gingivalis, Prevotella intermedia, Aggregatibacter actinomycetemcomitans, Fusobacterium nucleatum, Bacteroides forsythus, Capnocytophaga species, and Campylobacter rectus. ${ }^{1}$ Among major periodontal pathogens, $P$. gingivalis appears to be one of the principal etiological agents in the pathogenesis as well as the progression of the inflammatory episodes of the periodontal disease process. About $85.75 \%$ of samples of subgingival plaque extracted from chronic periodontitis patients established the role of this periodontopathic bacterium in this chronic disease process. ${ }^{2}$ 
Various research work directed towards the role of diode lasers in adjunctive to scaling and root planning have shown promising results in reduction of bacterial count especially specific periodontal pathogens like Porphyromanas gingivalis and Prevotella intermedia in cases of chronic periodontitis. ${ }^{3}$ Literature also reveals role of diode lasers in reducing gingival bleeding, reducing clinical attachment loss as well as decreasing tooth mobility. ${ }^{4}$ Henceforth, due to prevalence of such effects, i.e. their bactericidal action on periodontal pathogens, lasers present an effective way out to swap adjunctive use of antibiotics in periodontal therapy. ${ }^{5}$ Thus, the present study was carried out to investigate the bactericidal effect of gallium aluminium arsenide (GaAlAs) laser on photosensitized predominant periodontal pathogenic anaerobic organisms, i.e., Porphyromonas gingivalis and Prevotella intermedia.

\section{MATERIALS AND METHODS}

The present prospective study was carried out among 50 patients aged $30-55$ years reporting to the department for the treatment of periodontitis with pockets $\geq 5 \mathrm{~mm}$ depth. Patients with more than 20 natural teeth and suffering from a chronic form of periodontitis were included in the study. Ethical permission was obtained from the ethical committee of the institute and patients were enrolled after obtaining informed consent. Smokers, patients who have taken antibiotics within a period of last 3 months and those suffering from any systemic disease were excluded from the study. For sample analysis of $P$. intermedia and $P$. gingivalis, a subgingival plaque was collected from periodontal pockets $\geq 5 \mathrm{~mm}$ depth using sterile curettes under aseptic conditions. The sample was then immediately transported to microbiology laboratory using transport media, thioglycollate broth with vitamin $\mathrm{K}$ and hemin. After 72 hours growth of bacterial colonies was seen on culture plates.

$P$. intermedia was identified as brown to black pigmented colonies on blood agar. P. gingivalis were identified based on colony characteristics, pigmentation and fluorescence activity. It showed black-pigmented colonies on blood agar. P. intermedia forms circular, convex, 1-2 mm, shiny, gray colonies. P. intermedia fluoresce a dark red on exposure to a Wood's lamp (long-wave UV light). By colony morphology and Gram stain, Porphyromonas gingivalis tend to form smaller colonies and present as shorter rods or coccobacilli on Gram stain. Porphyromonas usually grows as pigmented colonies, initially forming gray colonies that darken to black colonies within a week after plating on blood agar. ${ }^{6}$ A predetermined number of colonies (for P. intermedia 500 colonies were taken initially; for $P$. gingivalis 400 colonies were taken initially) were taken equally as control group and case group (Fig. 1) Colony count was carried out and expressed as CFU/ML. Thirty $\mu \mathrm{L}$ of $100 \mu \mathrm{g} / \mathrm{mL}$ of Toluidine blue $\mathrm{O}$ dye was added, and the study group were exposed to GaAlAs diode laser (Fig. 1) with a power output of $10 \mathrm{Mw}$ at a wavelength $810 \mathrm{~nm}$ in a pulsed mode with a frequency of $20 \mathrm{kHz}$ for one minute, in non- contact mode. After irradiation bacterial colonies of study and control group were then serially diluted (in ThioGlycollate Broth) in 1:10. 1:50 and 1:100 ratio and subcultured under the same culture conditions and incubated for 72 hours. After incubation period again the number of the viable bacterial count was carried out using a magnifying glass and expressed as $\mathrm{CFU} / \mathrm{mL}$, to determine the bactericidal effect of GaAlAs laser. The data was statistically analyzed using the SPSS statistical software version 16. Mann-Whitney Test was used for statistical analysis with $p$ value less than 0.05 considered statically significant.

\section{RESULTS}

Table 1 shows the bactericidal effect of GaAlAs Laser on P. intermedia and P. gingivalis. Initially, 486 colonies of $P$. intermedia were taken as the control group which reduced to 394,287 , and 136 after dilution in ThioGlycollate Broth in 1:10, 1:50 and 1:100 ratios. Study group shows a reduction in colony count after being exposed to GaAlAs diode laser for 1 minute from 369 to 229,134 and 41 which was statistically significant with $p$ value $<0.0001$ (Graph 1).

Initially, 381 colony count of $P$. gingivalis were taken as the control group which reduced to 314, 215and 163 after dilution in Thio Glycollate Broth in 1:10, 1:50 and 1:100 ratios. Case group shows a decrease in colony count after being exposed to GaAlAs diode laser for 1 minute from 286.52 to 197 , 94and 39which was statistically significant with $p$ value $<0.0001$ (Graph 2).

\section{DISCUSSION}

Prevotella intermedia is a black-pigmented anaerobic gram-negative bacterium which is associated with oral diseases, mainly periodontitis. The hitch in eradicating the bacterium has been attributed to the resistance of $P$. intermedia to many antibiotics, including cephalosporins, penicillins as well as tetracyclines. Moreover, cells of $P$. intermedia forms a biofilm which protects bacterial cells and due to which it is more resistant to antibiotics. For the reason that biofilm can act as a reservoir of antibiotic resistance, it is of clinical significance to develop alternative antimicrobial approaches for controlling antibioticresistant $P$. intermedia. ${ }^{7}$ In the present study, study group showed reduction in colony count of $P$. intermedia after 

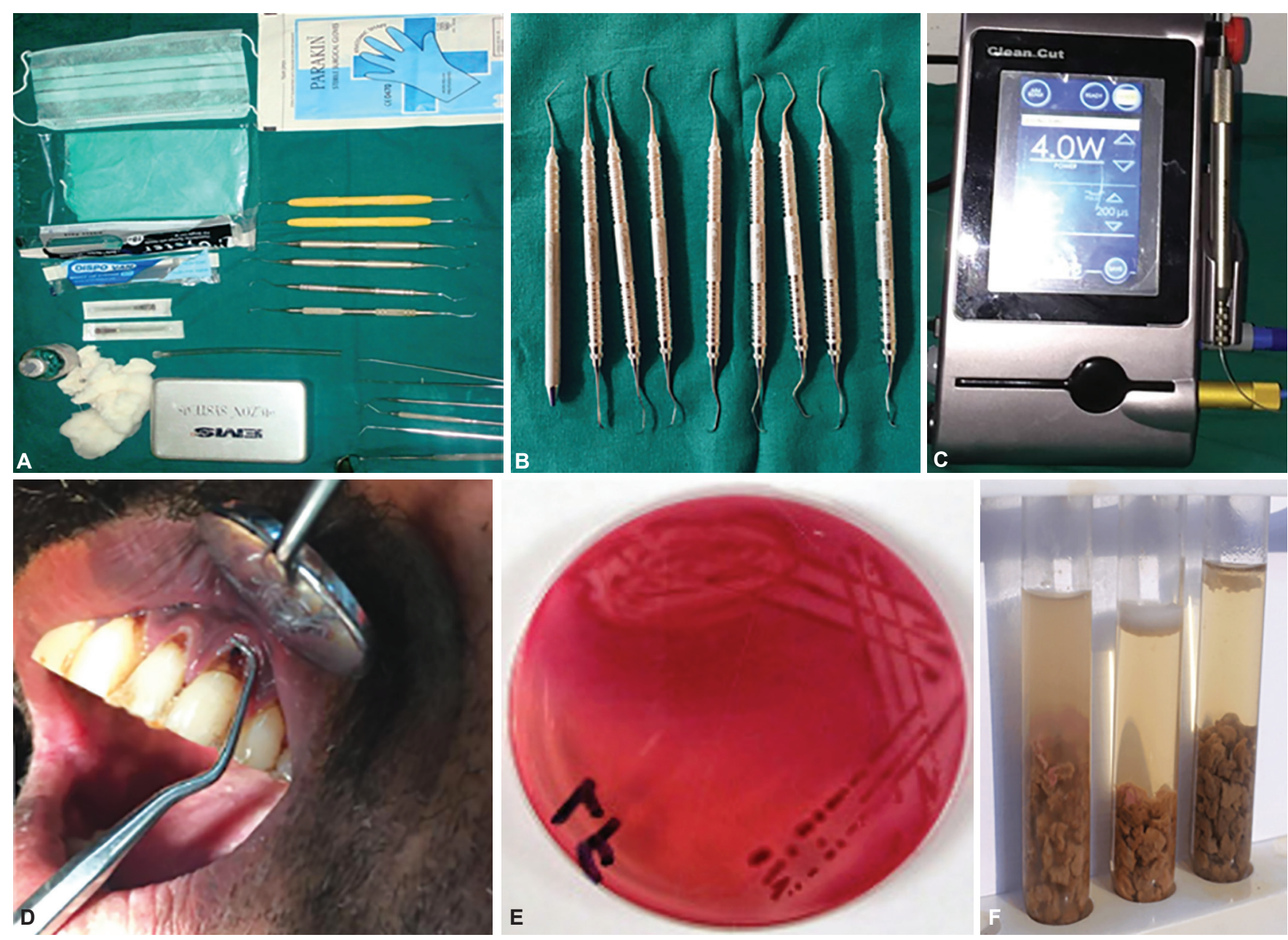

Figs 1A to E: (A); Armamentarium used in study; (B); Different curettes and scalers; (C); Diode laser; (D); Removal of plaque using curette; (E); Colonies on blood agar culture media; $(F)$ : Transport media, thioglycollate broth

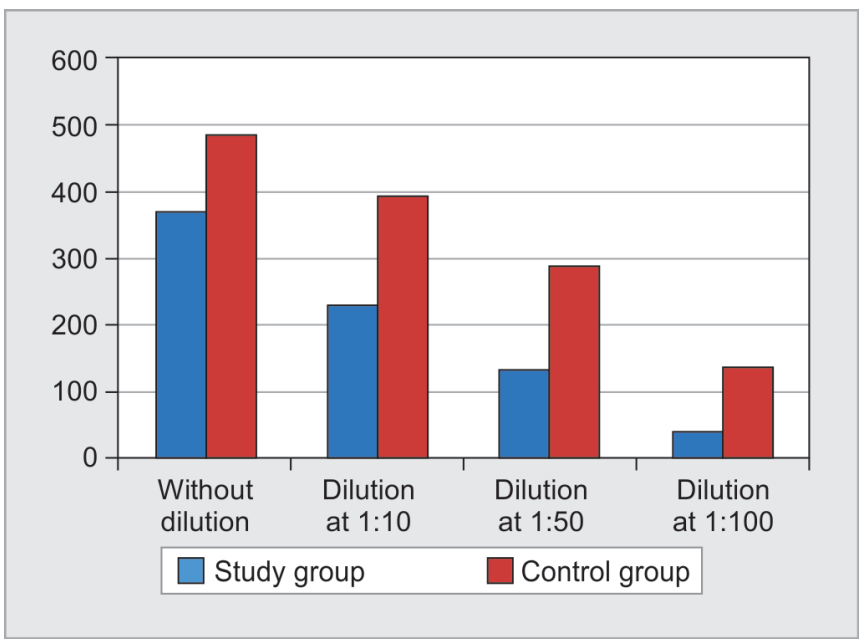

Graph 1: Bactericidal effect of GaAIAs laser on P. intermedia

being exposed to GaAlAs diode laser for 1 minute from 369 to 229, 134 and 41 after serial dilution of in ThioGlycollate Broth in 1:10, 1:50 and 1:100 ratios which was statistically significant with $p$ value $<0.0001$.

Porphyromonas gingivalis, an etiological agent in severe forms of periodontitis, is a prominent component of the

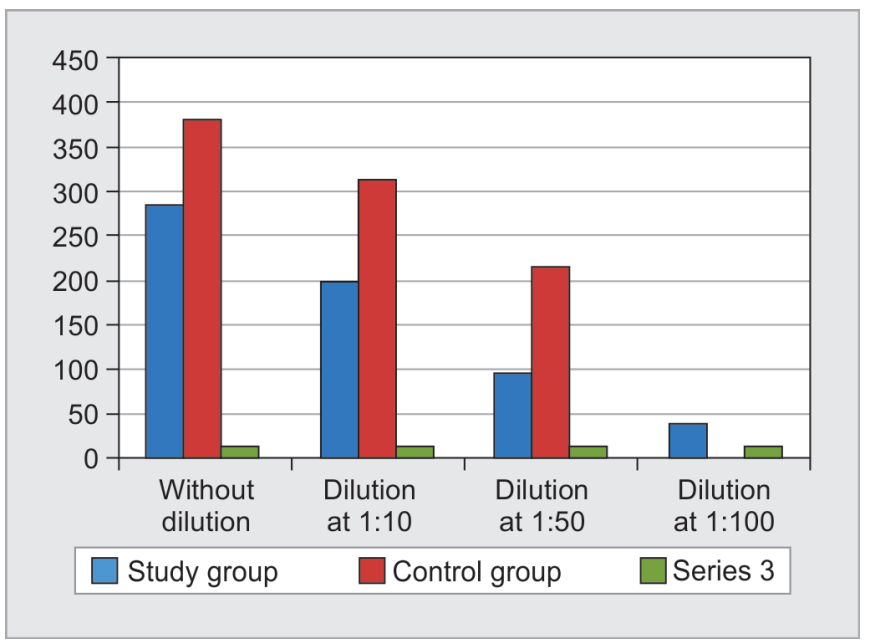

Graph 2: Bactericidal effect of GaAlAs laser on P. gingivalis

oral microbiome and a successful colonizer of the oral epithelium that plays role in alveolar bone resorption. ${ }^{8}$ Lipopolysaccharide of $P$. gingivalis circulates systemically in over $50 \%$ of periodontal disease patients and is associated with increased matrix metalloproteinase. ${ }^{9}$ It contains virulence factors such as lipopolysaccharide and 


\begin{tabular}{lcccc}
\hline & \multicolumn{2}{c}{ Table 1: Bactericidal Effect of GaAlAs Laser on P. intermedia and P. gingivalis } \\
\cline { 2 - 5 } & \multicolumn{3}{c}{ P. intermedia } & \multicolumn{2}{c}{ P. gingivalis } \\
\cline { 2 - 5 } & $369.0 \pm 24.48$ & $486.54 \pm 12.18$ & $286.52 \pm 10.54$ & $381 \pm 13.73$ \\
\hline Without Dilution & $229.48 \pm 39.52$ & $394.18 \pm 29.61$ & $197 \pm 12.47$ & $314 \pm 18.28$ \\
Dilution at 1:10 & $134.67 \pm 34.49$ & $287.0 \pm 32.64$ & $94 \pm 14.51$ & $215 \pm 16.94$ \\
Dilution at 1:50 & $41.54 \pm 26.42$ & $136.33 \pm 15.86$ & $39 \pm 10.29$ & $163 \pm 21.49$ \\
Dilution at 1:100 & & $<0.0001$ & & $<0.0001$ \\
$p$ value (Mann-Whitney Test) & &
\end{tabular}

gingipains. These virulence factors may contribute to tissue destruction in periodontal diseases by serving as a vehicle for the antigens and active proteases..$^{10}$ In the present study results showed a reduction in colony count of $P$. gingivalis from 381 to 314, 215 and 163 CFU/ML after dilution in ThioGlycollate Broth in 1:10, 1:50 and 1:100 ratios after being exposed to GaAlAs diode laser for 1 minute which was statistically significant.

Benachinmardi et al. ${ }^{11}$ studied the nature of oral microbiota in chronic periodontitis and reported that anaerobic bacteria are an important cause of chronic periodontitis, i.e., Fusobacterium spp, Bacteroides fragilis, Porphyromonas spp and Prevotella intermedia are the most common anaerobic pathogens along with aerobes and microaerophilic organisms.

Lasers are termed according to the active element(s) that is induced to undergo the stimulated quantum transitions that, consecutively, creates the energy beam. ${ }^{12}$ Specific indications for gallium-aluminium-arsenide (GaAlAs) laser are soft tissue incision and ablation; subgingival curettage; bacterial elimination, pulpotomy, aphthous ulcer treatment, analgesia, melanin pigment removal, root canal disinfection, sulcular debridement, caries removal, treatment of dentine hypersensitivity. ${ }^{13}$ What is more, among the various laser types with corresponding wavelengths, diode laser systems have established themselves as compact, economic and versatile, predominantly for performing soft tissue applications. Research has shown that near-infrared (NIR) wavelengths are ideally suited for numerous soft tissue procedures due to their high absorption in hemoglobin. Among the available NIR wavelengths, it has been found that the wavelengths around $810 \mathrm{~nm}$ to be one of the most versatile with regard to the number of possible treatment options, as this wavelength range can be effectively used in the field of soft tissue surgery, periodontics, endodontics, implantology, and tooth whitening. ${ }^{14}$ Hence, in the present studyaluminium, GaAlAs laser with wavelength $810 \mathrm{~nm}$ was used to evaluate its bactericidal property.

Methylene blue and toluidine blue $\mathrm{O}$ are very effective photosensitizing agents for the inactivation of both gram-positive and gram-negative periodontopathic bacteria. ${ }^{15}$ Dobson et al. ${ }^{16}$ demonstrated that bacteria associated with periodontal disease can be killed through photosensitization with toluidine blue $\mathrm{O}$ by irradiating with a helium-neon soft laser. Thus, in the present study toluidine blue $\mathrm{O}$ was used as a photosensitizer to evaluate the bactericidal action of GaAlAs laser.

Sakurai et al. ${ }^{17}$ indicated that irradiated cells produced less Prostoglandin E2 (PGE2) and Cycl-O-Oxynase (COX), indicating the possible anti-inflammatory role of the diode laser which may have a therapeutic effect on aggravation of periodontitis. Kreisler et al. ${ }^{18}$ demonstrated that the proliferative activity of ligament fibroblasts was considerably higher after $890 \mathrm{~nm}$ wavelength diode laser irradiation. Moritz et al. ${ }^{19,20}$ concluded that the diode laser treatment following scaling and root planning had a bactericidal effect and reduced inflammation. Identical antimicrobial results were obtained by Shibli et al. ${ }^{21}$ who reported that PDT could reduce the bacterial count of P. intermedia, P. nigrescens, Fusobacterium spp. in ligatureinduced peri-implantitis of dogs. Similarly, Luchesi et al. ${ }^{22}$ investigated the effect of photodynamic therapy as an adjunct to mechanical therapy in furcations. Photodynamic therapy showed advantages in local levels of cytokines and a reduction in periodontal pathogens was demonstrated. The findings of our study are also in agreement with Voos et al. ${ }^{23}$ who compared the antibacterial efficiency of a PDT using the photosensitizer safranine $\mathrm{O}$ with that of chlorhexidine $(0.2 \% \mathrm{CHX})$ on an ex vivo biofilm. Photodynamic treatment with safranine $\mathrm{O}$ showed a distinct antibacterial effect on F. nucleatum and P. gingivalis whereas S. gordonii was suppressed completely by a PDT, treatment with $2 \%$ CHX caused only a partial reduction.

In addition to the bactericidal effect of the laser technique, it is one of the methods used to hasten the recovery of tissue functionality in the treatment of wounded tissues as they improve the recovery of the tissue by inducing vessel dilatation, collagen synthesis and by increasing tissue oxygenation. ${ }^{24}$ Reddy ${ }^{25}$ in his comprehensive review regarding the role of lowintensity laser therapy (laser photostimulation) in biology and medicine reported gallium-arsenide (GaAs) laser among diabetic rats was found to be effective in healing wounds by promoting the amount of collagen significantly. Posten ${ }^{26}$ in a literature review revealed that GaAlAs laser (904 nm), when applied to the microcirculation of live rats, 
promotes dilatation of the irradiated arterioles, followed by an increase in arteriolar blood flow. Therefore, in addition, to bactericidal effect of laser therapy which is attributed to the elimination of lipopolysaccharide, ability to remove bacterial plaque and calculus and irradiation effect limited to an ultra-thin layer of tissue, moreover, its ability to hasten bone and soft tissue repair, make it a potential tool for periodontal treatment including scaling and root surface debridement. ${ }^{27}$

The limitation of the present study is that it is an in vitro study and therefore, research is recommended to further evaluate results by commencing in vivo studies to assess its clinical applicability.

\section{CONCLUSION}

The result of our study implies that for the tested bacterial strains, the effect of GaAlAs laser for 1 minute resulted in a significant reduction in the viable counts of periodontal pathogenic anaerobic organisms i.e., simplicity of the photodynamic therapy and effective bacterial eradication are the imperative reasons why PDT is being extensively becoming a research interest in periodontics. Further, it can thus be safely considered that this technique is an alternative method adjunct to mechanical therapy.

\section{REFERENCES}

1. Kesic L, Milasin J, Igic M, Obradovic R. Microbial Etiology of Periodontal Disease - Mini Review. Medicine and Biology 2008;15(1):1-6.

2. How KY, Song KP, Chan KG. Porphyromonas gingivalis: An Overview of Periodontopathic Pathogen below the Gum Line. Frontiers in Microbiology. 2016;7:53.

3. Yilmaz S, Kuru B, Kuru L, Noyan U, et al. Effect of gallium arsenide diode laser on human periodontal disease: A microbiological and clinical study. Lasers Surg Med 2002;30:60-66.

4. Coluzzi D. Fundamentals of dental lasers: science and instruments, D Clin N Am 2004;48:751-770.

5. Dae-hyun L. Application of Laser in Periodontics: A New Approach in Periodontal Treatment. Dental Bulletin The Hong Kong Medical Diary 2007;12(10):23-25.

6. Garrett WS, Onderdonk AB. Bacteroides, Prevotella, Porphyromonas, and Fusobacterium species (and other medically important anaerobic Gram-negative bacilli). In Mandell, Douglas, and Bennett's Principles and Practice of Infectious Diseases (Eighth Edition) 2015 (pp. 2773-2780).

7. Jang EY, Kim M, Noh MH, et al. In vitro effects of polyphosphate against Prevotella intermedia in planktonic phase and biofilm. Antimicrobial agents and chemotherapy. 2016 Feb 1;60(2):818-826.

8. Chan Y, Lai CH. Bactericidal effects of different laser wavelengths on periodontopathic germs in photodynamic therapy. Lasers in medical science. 2003 Mar 1;18(1):51-55.

9. Al Batran R, Al-Bayaty FH, Al-Obaidi MM. In-vivo effect of andrographolide on alveolar bone resorption induced by Porphyromonas gingivalis and its relation with antioxidant enzymes. BioMed research international. 2013;2013.
10. DeLeon-Pennell KY, de Castro Brás LE, Lindsey ML. Circulating Porphyromonas gingivalis lipopolysaccharide resets cardiac homeostasis in mice through a matrix metalloproteinase9-dependent mechanism. Physiological reports. 2013 Oct $1 ; 1(5)$.

11. Benachinmardi KK, Nagamoti J, Kothiwale S, et al. Microbial Flora in Chronic Periodontitis: Study at a Tertiary Health Care Center from North Karnataka. Journal of Laboratory Physicians 2015;7(1):49-54.

12. Verma SK, Maheshwari S, Singh RK, et al. Laser in dentistry: An innovative tool in modern dental practice. National journal of maxillofacial surgery. 2012 Jul;3(2):124.

13. Cobb CM. Lasers in Periodontics: A Review of the Literature J Periodontol 2006;77(4):545-564.

14. Pirnat S. Versatility of an $810 \mathrm{~nm}$ diode laser in dentistry: An overview. J Laser Health Acad. 2007;4(2):1-9.

15. Miskoski S, Sanchez E, Garavano M, et al. Singlet Molecular oxygen-mediated photo-oxidation of tetracyclines kinetics, mechanism and microbiological implications. J Photochem Phtobiol B - Biol. 1998;43:164-171.

16. Dobson J, Wilson M. Sensitization of oral bacteria in biofilms to killing by light from a low-power laser. Arch OralBiol. 1992;37:883-887.

17. Sakurai Y, Yamaguchi M, Abiko Y. Inhibitory effect of lowlevel laser irradiation on LPS-stimulated prostaglandin E2 production and cyclooxygenase-2 in human gingival fibroblasts. Eur J Oral Sci. 2000; 108(1):29-34.

18. Kreisler M, Christoffers AB, Willershausen B, et al. Effect of low-level GaAlAs laser irradiation on the proliferation rate of human periodontal ligament fibroblasts: an in vitro study. J Clin Periodontol. 2003; 30(4):353-358.

19. Moritz A, Gutknecht N, Doertbudak O, et al. Bacterial reduction in periodontal pockets through irradiation with diode laser: a pilot study. J Clin Laser Med Surg. 1997; 15(1): 33-37.

20. Moritz A, Schoop U, Goharkhay K, et al. Treatment of periodontal pockets with diode laser. Lasers Surg Med. 1998; 22(5):302-311.

21. Shibli JA, Martins MC, Theodoro LH, et al. Lethal photosensitization in microbiological treatment of ligature-induced peri - implantitis: A preliminary study in dogs. J Oral Sci.2003;45:17-23.

22. Luchesi VH, Pimentel SP, Kolbe MF, et al. Photodynamic therapy in the treatment of class II furcation: a randomized controlled clinical trial. J Clin Periodontol. 2013 Aug;40(8):781-788.

23. Voos AC, Kranz S, Tonndorf-Martini S, et al. Photodynamic antimicrobial effect of safranine $\mathrm{O}$ on an ex vivo periodontal biofilm. Lasers Surg Med. 2014 Mar;46(3):235-243.

24. Gonçalves RV, Mezêncio JM, Benevides GP, et al. Effect of gallium-arsenide laser, gallium-aluminum-arsenide laser and healing ointment on cutaneous wound healing in Wistar rats. Brazilian J Med Biol Res. 2010 Apr;43(4):350-355.

25. Reddy GK. Photobiological basis and clinical role of lowintensity lasers in biology and medicine. J Clin Laser Med Surg 2004;22:141-150.

26. Posten W, Wrone DA, Dover JS, et al. Low-level laser therapy for wound healing: mechanism and efficacy. Dermatologic surgery. 2005 Mar;31(3):334-340.

27. Dang AB, Rallan NS. Role of lasers in periodontology: A Review. Annals. 2013 Jul 1;1(1):8. 